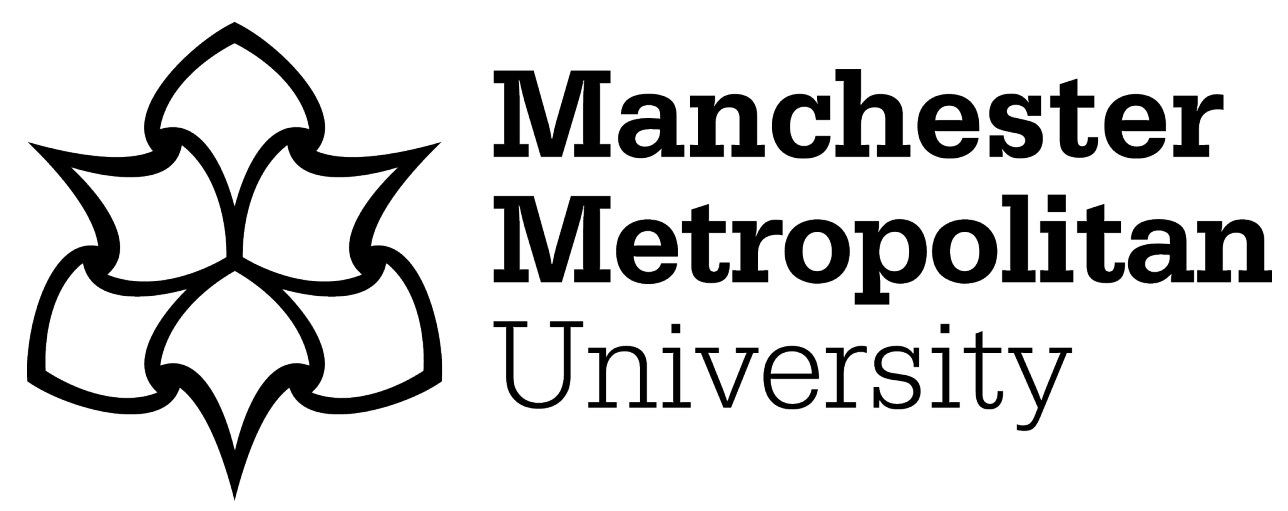

Landreth, K, Simanaviciute, U, Fletcher, J, Grayson, B, Grant, Robyn, Harte, $\mathrm{MH}$ and Gigg, J (2021) Dissociating the effects of distraction and proactive interference on object memory through tests of novelty preference. Brain and Neuroscience Advances, 5. p. 1. ISSN 2398-2128

Downloaded from: https://e-space.mmu.ac.uk/627588/

Version: Published Version

Publisher: Sage

DOI: https://doi.org/10.1177/23982128211003199

Usage rights: Creative Commons: Attribution 4.0

Please cite the published version 


\title{
Dissociating the effects of distraction and proactive interference on object memory through tests of novelty preference
}

Brain and Neuroscience Advances Volume 5: 1-11

(c) The Author(s) 2021

Article reuse guidelines:

sagepub.com/journals-permissions DOI: $10.1177 / 23982128211003199$ journals.sagepub.com/home/bna

@SAGE

\author{
K. Landreth ${ }^{*}$, U. Simanaviciute $1,2 *$, J. Fletcher ${ }^{3}$, B. Grayson ${ }^{3}$, \\ R. A. Grant ${ }^{2}$, M. H. Harte ${ }^{3}$ and J. Gigg ${ }^{1}$ iD
}

\begin{abstract}
Encoding information into memory is sensitive to distraction while retrieving that memory may be compromised by proactive interference from preexisting memories. These two debilitating effects are common in neuropsychiatric conditions, but modelling them preclinically to date is slow as it requires prolonged operant training. A step change would be the validation of functionally equivalent but fast, simple, high-throughput tasks based on spontaneous behaviour. Here, we show that spontaneous object preference testing meets these requirements in the subchronic phencyclidine rat model for cognitive impairments associated with schizophrenia. Subchronic phencyclidine rats show clear memory sensitivity to distraction in the standard novel object recognition task. However, due to this, standard novel object recognition task cannot assess proactive interference. Therefore, we compared subchronic phencyclidine performance in standard novel object recognition task to that using the continuous novel object recognition task, which offers minimal distraction, allowing disease-relevant memory deficits to be assessed directly. We first determined that subchronic phencyclidine treatment did not affect whisker movements during object exploration. Subchronic phencyclidine rats exhibited the expected distraction standard novel object recognition task effect but had intact performance on the first continuous novel object recognition task trial, effectively dissociating distraction using two novel object recognition task variants. In remaining continuous novel object recognition task trials, the cumulative discrimination index for subchronic phencyclidine rats was above chance throughout, but, importantly, their detection of object novelty was increasingly impaired relative to controls. We attribute this effect to the accumulation of proactive interference. This is the first demonstration that increased sensitivity to distraction and proactive interference, both key cognitive impairments in schizophrenia, can be dissociated in the subchronic phencyclidine rat using two variants of the same fast, simple, spontaneous object memory paradigm.
\end{abstract}

\section{Keywords}

Schizophrenia, object memory, preclinical model, phencyclidine, novel object recognition, whisker movement, parvalbumin, phencyclidine

Received: 8 December 2020; accepted: 22 February 2021

\section{Introduction}

The encoding of new information into memory depends on being able to stay 'on track', that is, to use focused attention to resist extraneous distraction. Retrieval of successfully encoded new memories then requires the ability to prevent proactive interference, that is, contagion from other, similar memories, disrupting current memory representations and decision-making (Postle and Brush, 2004; Postle et al., 2004). Sensitivity to both of these factors is a core symptom of several neuropsychiatric states, including early Alzheimer's disease (Bondi et al., 1994). Indeed, cognitive impairments associated with schizophrenia (CIAS) include cognitive control (Nuechterlein et al., 2004) and its core executive function of inhibitory control, which includes focussed attention (resistance to distraction) and cognitive inhibition (Diamond, 2013). Impaired resistance to proactive interference may be an underlying factor for a variety of memory impairments in schizophrenia (Burton et al., 2018; Ettinger et al., 2018; Girard et al., 2018; Heckers et al., 2000; Park et al., 2003). These symptoms are severely debilitating (Elvevag and Goldberg, 2000; Green, 1996), occur prior to psychosis (Kahn and Keefe, 2013) and are resistant to treatment (Keefe et al., 2007; Nuechterlein et al., 2004). The CNTRICS initiative identified

${ }^{1}$ Division of Neuroscience and Experimental Psychology, Faculty of Biology, Medicine and Health, The University of Manchester, Manchester, UK

2Department of Natural Sciences, Manchester Metropolitan University, Manchester, UK

${ }^{3}$ Division of Pharmacy, Faculty of Biology, Medicine and Health, The University of Manchester, Manchester, UK

${ }^{*}$ Equal contribution.

Corresponding author:

J. Gigg, Division of Neuroscience and Experimental Psychology, Faculty of Biology, Medicine and Health, The University of Manchester, Oxford Road, Manchester M13 9PT, UK.

Email: j.gigg@manchester.ac.uk 
attention and cognitive inhibition as symptom areas with high translational potential for preclinical research (Barch et al., 2009) and several preclinical, operant-based tasks with translational validity have been developed to meet this need (Gilmour et al., 2013). However, while these tasks highlight rule-based, attentional and interference features, they also commonly require weeks to months of training to attain threshold performance. Ideally, our preclinical task armoury to investigate distraction and interference effects should also include high-throughput, simple, short-duration tasks that require only spontaneous behaviour.

The novel object recognition (NOR) paradigm has been used most commonly to characterise the subchronic phencyclidine (scPCP) model of CIAS (Cadinu et al., 2018; Lisman et al., 2008; Neill et al., 2010, 2014). The standard novel object recognition task (stNOR) depends on maintaining object information in short-term memory and scPCP treatment induces an NOR deficit in mice (Gigg et al., 2020; Hashimoto et al., 2008; Nagai et al., 2009) and rats (Horiguchi and Meltzer, 2012; Snigdha et al., 2010) that is produced by distraction during the inter-trial interval (Gigg et al., 2020; Grayson et al., 2014). This supports the conclusion that, while the scPCP model can encode object memory, maintaining this information is abnormally sensitive to disruption. While this demonstrates the cross-species validation of a simple and fast means to measure distraction susceptibility, this effectively precludes stNOR as a probe for disease-relevant memory deficits. However, recent developments allow NOR to be probed in a continuous trial design (conNOR) that allows the sequential performance of 10 or more object preference trials in a single session, all with minimal distraction between task phases (Albasser et al., 2010; Ameen-Ali et al., 2012; Chan et al., 2018). Using such an approach provides a promising means to probe object memory deficits independent of distraction in the scPCP and other models of human neuropsychiatric conditions.

Here, we first ensured that scPCP treatment had no a priori effect on object sensing through whisker movements. This was important as whisker kinematics provide key tactile information to rodents about the environment and are altered in other rodent disease models (Grant et al., 2014, 2020; Simanaviciute et al., 2020). We next ran stNOR to reproduce the effect of distraction in scPCP rats. Following this, rats experienced 11 trials of a single conNOR session to test the effect of proactive interference on memory performance without the potential confound of distraction. Our hypothesis was that we could achieve behavioural dissociation for the effects of distraction and proactive interference on object memory by comparing scPCP performance between these two-related, simple, spontaneous tasks. A positive result here would add further relevance to the scPCP model for preclinical research and provide attractively simple and high-throughput methods to probe high-level cognitive deficits relevant to schizophrenia.

\section{Methods}

\section{Animals}

Experiments were performed using 20 female Lister hooded rats (Charles River, UK; 190-224 g at study start). Our previous work has shown that such group sizes are sufficient to reveal a significant behavioural phenotype for object recognition in scPCPtreated rats that is also sensitive to disruption (Grayson et al.,
2014). Animals were housed in groups of five in standard housing conditions (Tecniplast ventilated cages, temperature $20^{\circ} \mathrm{C} \pm 2{ }^{\circ} \mathrm{C}$ and humidity $55 \% \pm 5 \%$, University of Manchester Biological Services Facility) with ad libitum access to standard chow and water. All experimental procedures were carried out in the light phase of their cycle (09:00-14:00) and performed under Home Office UK project licence in accordance with the Animals (Scientific Procedures) Act UK 1986 and approved by the University of Manchester AWERB (Animal Welfare and Ethical Review Body). A summary of all experimental stages is provided in Figure 1(a).

\section{Dosing}

Ten rats were dosed with phencyclidine hydrochloride $(2 \mathrm{mg} / \mathrm{kg}$, i.p.; scPCP) and the other 10 with vehicle ( $0.9 \%$ saline, i.p.). Dosing for all rats followed a standard subchronic regimen with injections delivered twice daily for 7 days, followed by a 7-day washout period (Grayson et al., 2014).

\section{Whisker movements}

Whisker movements were filmed after dosing to determine whether scPCP treatment changed the way the rats explored objects. Testing was carried out as described previously (Grant et al., 2014; Simanaviciute et al., 2020). Rats explored two different objects sequentially ( $5 \mathrm{~min}$ each) for one $10 \mathrm{~min}$ session in a $30 \mathrm{~cm} \times 50 \mathrm{~cm} \times 15 \mathrm{~cm}$ transparent Perspex enclosure sitting on a light box $(59.4 \mathrm{~cm} \times 84.1 \mathrm{~cm})$. Objects $(14.3 \mathrm{~cm} \times 6 \mathrm{~cm} \times 3 \mathrm{~cm})$ were composed of three smooth plastic toy bricks with $50 \%$ of these covered in tape to provide a different texture. The box and objects were cleaned with $70 \%$ ethanol between rats. Whisker movements were filmed at 500 frames per second by an overhead high-speed video camera (Phantom Miro ex2, resolution $640 \times 480$ pixels). Video clips were collected for each rat by manual trigger when it moved towards an object. Clips were trimmed and included for analyses if they fit published criteria where: (1) the rat was clearly in frame, (2) whiskers on both sides of the face were visible, (3) the head was parallel to the floor (no extreme pitch or yaw, no object climbing), (4) there were at least 50 frames of contact with the object (no contact with the walls of the box) and (5) the tracked portion of the clip was at least 150 frames long (Grant et al., 2014). Since this investigation was only concerned with object exploration, this was defined as the rat contacting the object with their whiskers, with no additional whisker contact on the walls of the box. After clip selection, 1-8 clips/rat, 149-1363 frames/clip, 0.298-2.726 s/clip were analysed using the Automated Rodent Tracker v2 (Gillespie et al., 2019). Image analysis located the tip of the rodent's nose and the body centroid (Figure 2). A co-registered scale bar provided a calibrated measure of locomotion speed. For whisker tracking, the software automatically found the snout orientation and position and the whisker angles relative to cranial midline (Figure 2). Whisker angles were measured as the angle between whisker and midline of the nose and head; larger angles represented more forward-positioned whiskers. Tracking was validated by manual inspection of video footage. Clips were removed where the tracked portion of the clip was less than 100 frames long, leaving 233 clips (106 vehicle and 127 scPCP, 119 smooth and 114 textured, 112 before and 121 after injections) for analysis. 
(a)

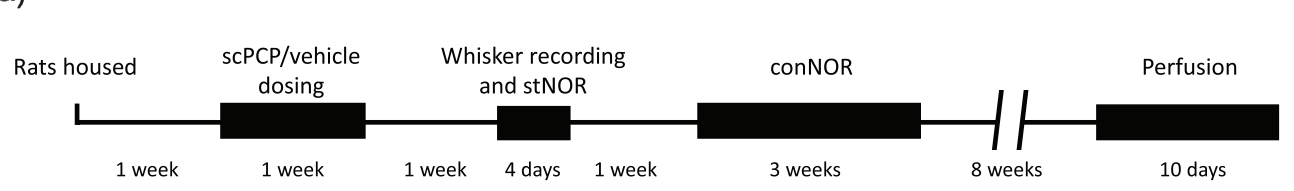

(b)

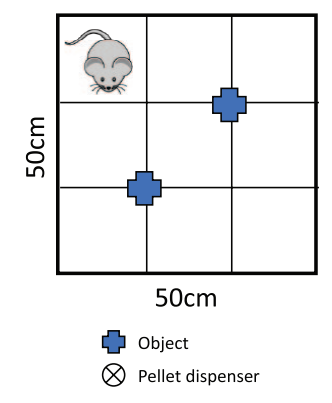

(c)

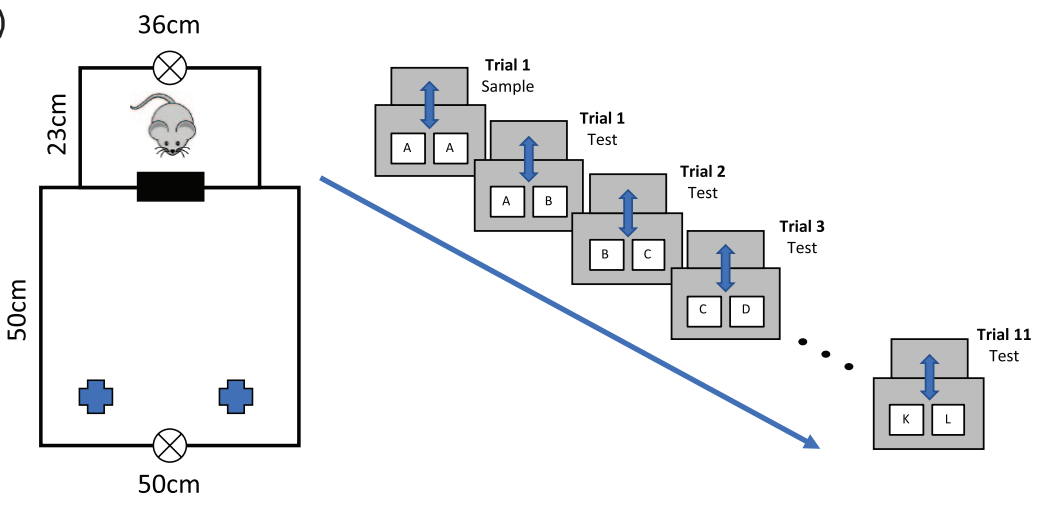

Figure 1. Experimental design. (a) Timeline for the study. (b) The stNOR arena. (c) The arena (left) and the trial design (right) for conNOR testing. Different pairs at test are displayed as $\mathrm{A}+\mathrm{A}, \mathrm{A}+\mathrm{B}$ and so on.

Mean whisker angle was calculated as a frame-by-frame average of all tracked whiskers on each side of the face. The mean whisker angles allowed the tracker to calculate the following whisker position and movement parameters: mean angular position (the average whisker angle, measured in degrees), amplitude $(2 \sqrt{ } 2 *$ the standard deviation of whisker angles, to approximate the range of whisker movements in degrees), asymmetry (the difference in the mean angular position between all whiskers on left and right sides; degrees) and the mean angular retraction and protraction speeds (calculated as the average speed of all the backward (negative) and forward (positive) whisker movements, respectively; degrees/s) (Gillespie et al., 2019). For mean angular position, amplitude and retraction and protraction speeds, the values for right and left whisker measurements were averaged.

\section{Object memory assessments}

\section{stNOR memory test}

After the 7-day dosing washout period, animals were subjected to a single NOR trial (Ennaceur and Delacour, 1988) to ensure that scPCP rats expressed the expected NOR deficit (Grayson et al., 2007). The NOR arena was a $50 \mathrm{~cm}^{3}$ box marked into a $3 \times 3$ grid on the floor (Figure 1(b)). A separate holding box was positioned close to the apparatus, consisting of a $15 \mathrm{~cm} \times 20 \mathrm{~cm}$ opaque plastic tub into which animals were placed during the inter-trial interval. Rats were first habituated to the test arena by placing them individually into the box for $15 \mathrm{~min}$. The next day, rats were subject to one stNOR session consisting of a 3-min object acquisition phase (explore two identical copies of a novel object), removal to the holding box for a 1-min inter-trial interval (ITI) then return to the test arena for a final 3-min test phase (explore a copy of the acquisition object and one novel object). Objects were well validated for equal baseline preference in prior testing, and we chose to adopt this task design here so that our approach would be directly comparable to our previous studies (Grayson et al., 2007). Objects were placed at positions equidistant from where the rat was introduced to the arena to ensure no spatial bias. The number of object visits and duration of object exploration was recorded via overhead video camera and analysed offline using the Novel Object Recognition Task Timer (https:// jackrrivers.com/program/). Rats were considered to be exploring when their nose was pointed towards and within $2 \mathrm{~cm}$ of an object; however, any time spent climbing on the object was discounted. The discrimination index (DI) between novel and familiar objects at test (stNOR) was measured by calculating the difference between exploration time for the two objects at test and dividing the result by the sum of their exploration time. Therefore, the more positive the value, the more exploration of the novel object at test, with negative values representing enhanced exploration of the familiar object.

\section{conNOR memory test}

The conNOR apparatus consisted of a holding chamber attached to a larger experimental arena by a computer-controlled sliding door (Campden Instruments Ltd., UK). An overhead video camera recorded exploration within the experimental chamber. Computer-controlled pellet dispensers with reward troughs were placed in both chambers on the walls furthest from the door (Figure 1(c)) to deliver standard rodent tablets (LabTab AIN76A, $45 \mathrm{mg}$; TestDiet, USA). Task sequences and operation of the door, pellet dispensers and recording of behavioural video and visits to retrieve pellets were managed and recorded by ABET II software (Campden Instruments Ltd.). The advantage of this arena for present purposes is that it does not require any handling 


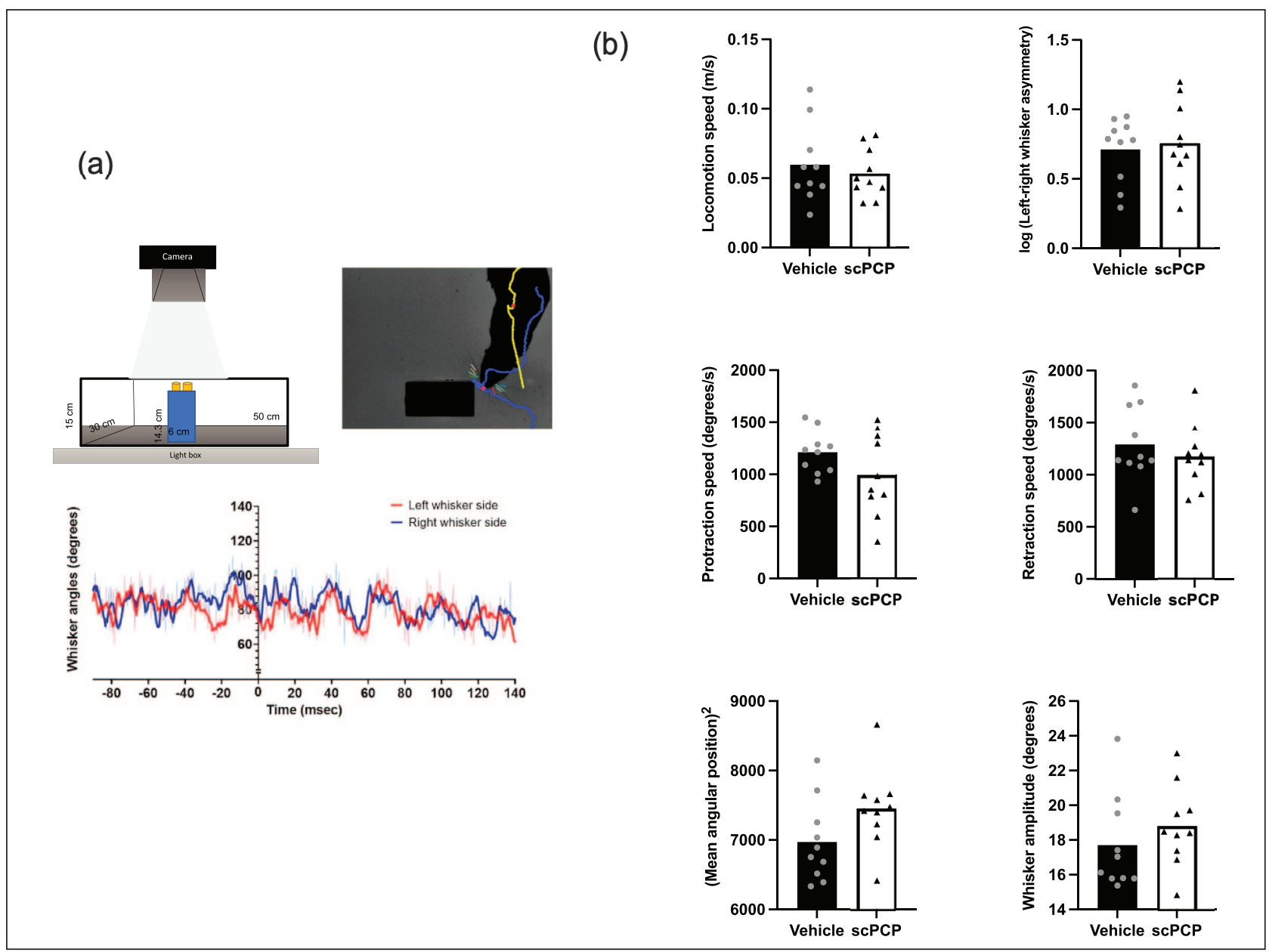

Figure 2. Whisker movement in SCPCP and vehicle rats. (a) Top left panel: diagram of object exploration arena; top right panel: video frame taken from typical whisker contact with an object to show tracking of whiskers, tip of nose (blue) and body centroid (yellow); bottom panel: example of whisker tracking; object contact occurred at time $x=0$; therefore, whisker measurements were extracted from after this time. (b) ScPCP and vehicle groups were similar in terms of: general locomotion speed in the arena, asymmetry of whisker position; whisker protraction and retraction speed; mean angular whisker position; and whisker amplitude. $N=10$ animals for both vehicle and SCPCP groups, with data presented as individual values plotted over group mean.

during the ITI; animals are only handled twice, first at the start and second for final removal once all trials have finished. Here, we kept to the training and testing protocol for conNOR developed by Chan et al. (2018) as this produced robust effects.

Rats were first habituated to the conNOR arena over 4 days, during which timed dispensing of food pellets encouraged shuttling between holding and experimental chambers (Chan et al., 2018). On day 1, cage groups were placed into the arena with the central door open for $30 \mathrm{~min}$ to explore freely, encouraged by single pellet dispensing into both chambers every minute. On day 2 , rats were placed singly into the holding chamber with the gate open for $20 \mathrm{~min}$ of free exploration with pellets delivered to both chambers every $1 \mathrm{~min}$. Animals that fed in and explored both chambers moved to habituation day 3 where they were placed in the holding chamber, a pellet was delivered and, when taken from the dispenser, the door opened and a further pellet was delivered in the experimental chamber. Once the animal shuttled successfully the door closed, opening again once the pellet had been taken from the experimental dispenser. This process continued for $20 \mathrm{~min}$, with animals moving on to habituation day 4 if they shuttled quickly between chambers at least 18 times. On habituation, day 4 rats were placed individually into the holding chamber and two identical objects were placed in the experimental chamber. After taking a pellet and shuttling to the experimental chamber, the rat could explore these objects for $5 \mathrm{~min}$ after which the door re-opened and a pellet was delivered to the holding chamber. Once the rat returned to the holding chamber for a $1 \mathrm{~min}$ ITI, the door closed and objects in the experimental chamber were exchanged for a second pair of novel objects (if the animal failed to re-enter the holding chamber within $3 \mathrm{~min}$ it was moved back to the habituation day 3 protocol). This protocol was repeated for a third pair of objects, with animals being required to actively explore the objects to be permitted to move on to the testing phase.

Rats were mildly food restricted on the evening prior to testing (12 g/rat/day standard chow) to encourage performance in the conNOR arena the next day. Animals were placed in the holding chamber and a pellet was delivered. Once the pellet was taken 
from the dispenser, the door opened and 1 min later a pellet was dispensed in the experimental chamber (apart from taking a pellet to initiate the testing sequence they were not required to retrieve or consume pellets at any other point during testing). The door closed once the rat had shuttled to the experimental chamber, which now contained two identical novel objects $(A+A)$. Objects in all trials were all of a similar in size and constructed of plastic, glass or ceramic. Rats explored the A objects for $2 \mathrm{~min}$ before the door re-opened and a pellet was delivered to the holding chamber, prompting the animal to shuttle and the door to close. During a 1-min ITI, the objects were removed and replaced with an identical object A and a novel object B. At the end of the ITI, the door re-opened and a pellet was delivered to the experimental chamber, permitting a further 2-min object exploration period. This process of shuttling between holding and experimental chambers was repeated over 11 object pairs $(\mathrm{A}+\mathrm{A}$, $\mathrm{A}+\mathrm{B}, \mathrm{B}+\mathrm{C}, \ldots \mathrm{K}+\mathrm{L}$; Figure $1(\mathrm{c})$ ) to allow for continuous testing of object memory. The side of the chamber on which the novel object was placed was counterbalanced within rats, along with the object order between rats. Object exploration and the number of visits to objects were measured from recorded video as per stNOR. For analyses, the DI metric was again used for each trial where exploration times were summed for all trials to that point. For example, for conNOR trial 3, the sum of exploration durations for familiar objects on trials 1-3 was subtracted from the sum of novel object exploration over the same trials, the result divided by the sum of these two measures (Chan et al., 2018). Additional behavioural parameters were extracted using an ABET analysis schedule from events recorded during each trial. These were used to estimate the motivation of all rats to perform the conNOR task and included the following: the latency to collect a food pellet from the dispenser in either the holding or experimental arena (first visit), the total number of entries to each pellet dispenser, the latency to cross between chambers upon door opening and the time to complete each trial.

\section{Tissue collection and immunohistochemistry}

Rats were anaesthetised with isoflurane and then perfused transcardially with phosphate buffered saline (PBS). The brains were collected, cut in the coronal plane to provide blocks that included medial prefrontal cortex (mPFC) (prelimbic and infralimbic cortices) and post-fixed in $4 \%$ formaldehyde for $72 \mathrm{~h}$ at $4{ }^{\circ} \mathrm{C}$. Once fixed, brains were immersed in $30 \%$ sucrose until they sunk and then stored at $-80^{\circ} \mathrm{C}$ until sectioning. Sections were cut at $30 \mu \mathrm{m}$ using a cryostat (Leica Biosystems, UK). One in four serial sections of the prefrontal cortex were suspended in cryoprotectant (30\% ethylene glycol, $30 \%$ glycerol, $10 \%$ PBS and $30 \% \mathrm{dH}_{2} \mathrm{O}$ ) and stored at $-20^{\circ} \mathrm{C}$ until stained. Sections were washed three times in PBS for 5 min each and then transferred into a hydrogen peroxide solution for $30 \mathrm{~min}\left(0.6 \% \mathrm{H}_{2} \mathrm{O}_{2}, 0.1 \%\right.$ Triton $\mathrm{X}-100$, $8.8 \%$ PBS, $10 \%$ methanol and $80.5 \% \mathrm{dH}_{2} \mathrm{O}$ ). The sections then underwent PBS wash for $5 \mathrm{~min}$ followed by protein block for $1 \mathrm{~h}$ (5\% normal horse serum, $0.4 \%$ Triton X-100 and 94.6\% PBS). Incubation was then started with parvalbumin (PV) primary antibody (1:5000; Swant, Switzerland) diluted in protein block solution at $4^{\circ} \mathrm{C}$ for $36 \mathrm{~h}$.

After incubation, samples were washed twice in PBS and incubated for $2 \mathrm{~h}$ with secondary antibody (biotinylated antimouse; 1:200; Vector Laboratories, UK; in protein block solution) and washed again in PBS. Sections were then incubated with a Vectastain ABC kit (Vector Laboratories), in the dark, for $2 \mathrm{~h}$. After a final PBS wash, samples were visualised using DAB substrate kit (Vector Laboratories). Samples were incubated for up to $15 \mathrm{~min}$ and transferred into distilled water to stop DAB staining. Sections were mounted onto slides and left to dry overnight. Samples were dehydrated using increasing concentrations of ethanol $(70 \%, 90 \%$ and $100 \%)$ followed by Histoclear ( $5 \mathrm{~min})$ and allowed to dry for $30 \mathrm{~min}$ then mounted using DPX (SigmaAldrich, UK).

Images were viewed on an Olympus BX51 microscope and analysed using Image-Pro Plus (v6.3.0.512, Media Cybernetics, Inc., USA). For each section, the region of interest (prefrontal cortex (PFC)) was delineated manually. We then used a twodimensional (2D) stereological method whereby 35 randomly selected field of views were analysed within this region at $20 \times$ magnification using a motorised stage. Within each field, a square counting box with inclusion and exclusion lines was used to determine the number of PV-positive interneurons (each box was $120 \mu \mathrm{m} \times 120 \mu \mathrm{m}$ ). The data are presented as cell density per $\mathrm{mm}^{2}$. All analyses were conducted with the experimenter blinded to treatment condition.

\section{Data collection and statistical analysis}

Videos of whisker movement were tracked and object exploration scored when still blind to treatment condition. All data were analysed by either the unpaired two-tailed Student's $t$-test or mixed analysis of variance (ANOVA) (with Geisser-Greenhouse correction where required) followed by post hoc comparisons (Sidak). All analyses were performed using the GraphPad Prism (v9.0).

\section{Results}

\section{Whisker movement assessment}

We tested whether scPCP treatment had any effect on whisker movement by filming object encounters for all rats after dosing. First, there was no effect of object texture on our measurements, so data from both object types were combined. As can be seen in Figure 2, for these combined measures, we observed no differences in whisker movements between vehicle and scPCP-treated rats (two-tailed unpaired $t$-test). Overall, these results show scPCP treatment has no effect on how rats explore objects through active whisker movement.

\section{Object memory testing using the stNOR paradigm}

During the initial acquisition phase, there were no differences in total exploration time between vehicle and scPCP groups; the exploration time for each of the two identical objects at acquisition was also similar both between objects and treatment groups (data not shown). At test, the mean number of object visits between groups was slightly higher in scPCP rats, but this difference was not significant (Figure S1(a)). When comparing exploration times of novel versus familiar objects at test (Figure 3(a)), there was a significant effect of object novelty $(F(1,18)=10.53$, 


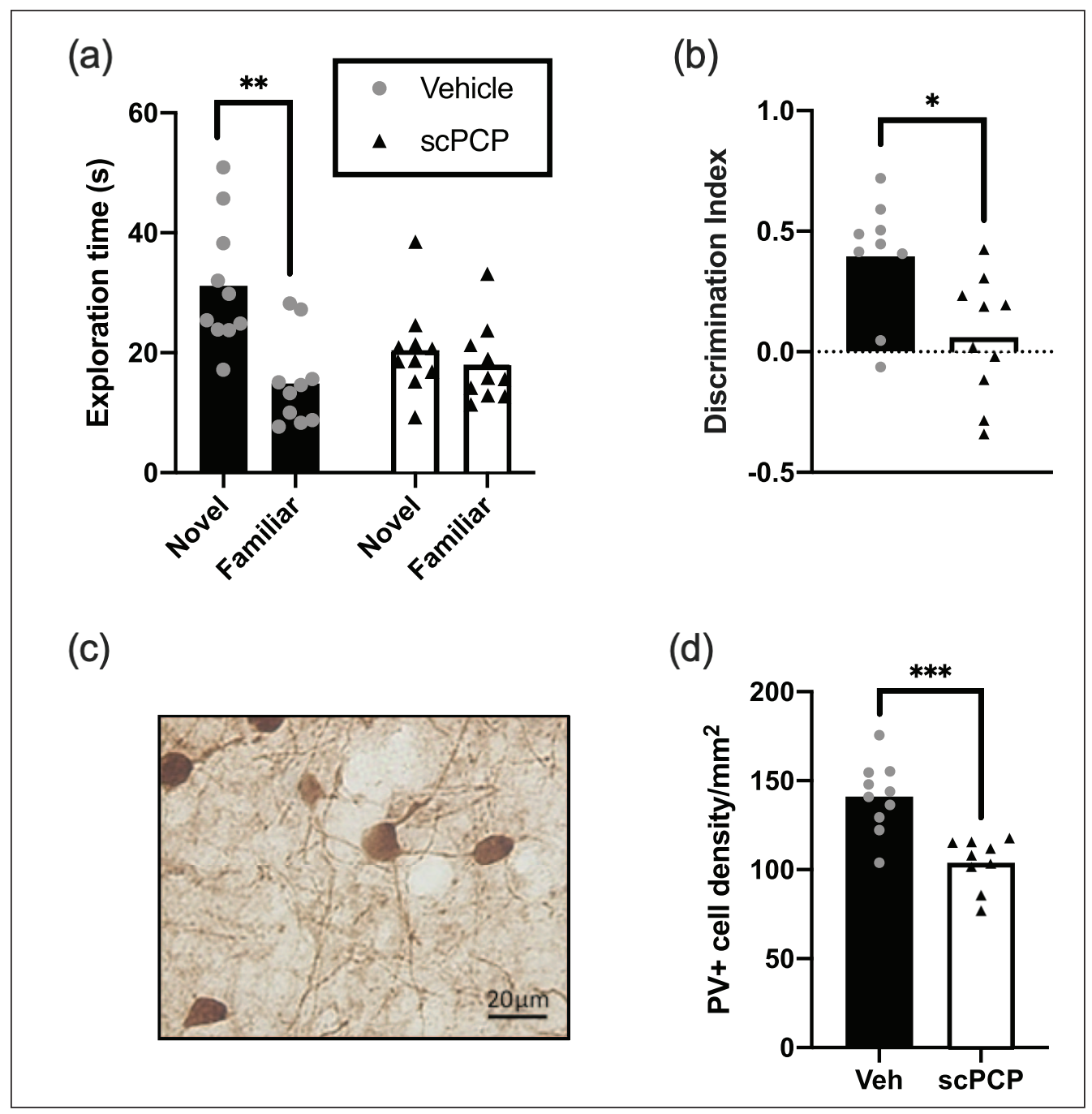

Figure 3. Standard NOR (stNOR) and PV deficits in SCPCP rats. (a) Exploration times for novel and familiar objects during stNOR test; only vehicle rats show a preference for the novel object with SCPCP rats exploring both novel and familiar objects similarly. (b) stNOR test performance plotted as discrimination index (DI); vehicle rats show significantly higher DI compared to SCPCP. Only vehicle group performance was greater than chance. (c) Typical example of parvalbumin staining from rat mPFC. Image $\times 20$. (d) The density of cells positively stained with anti-parvalbumin antibody (PV+) is lower in SCPCP group. $N=10$ for both vehicle and SCPCP groups in all panels with their data presented as individual values plotted over group mean. ${ }^{*} p<0.05 ;{ }^{* *} p<0.01 ;{ }^{* *} p<0.001$.

$p<0.01)$ and interaction between object novelty and group $(F(1,18)=5.666, p<0.05)$ with post hoc tests revealing significantly higher exploration of novelty in vehicle-treated rats only $(p<0.01$, Sidak). This result was also seen when comparing exploration as DI preference score (Figure 3(b)) with vehicle rats showing a significantly higher DI score compared to scPCP $(p<0.05$, unpaired $t$-test) and only vehicle animals showing DI scores above chance $(\mathrm{DI}=0 ; p<0.001$, one sample $t$-test). Overall, these results show that ScPCP rats displayed the expected stNOR deficit (Grayson et al., 2007).

\section{Object memory testing using a conNOR paradigm}

We first compared both groups' general operant performance over conNOR trials to determine whether food restriction had any differential effect on scPCP rats (Figure S2). We found no significant difference in any measure, supporting the view that vehicle and scPCP rats were equally motivated to perform the conNOR task. Similar to the stNOR, the mean number of object visits between groups over all trials in conNOR was slightly higher in scPCP rats, but this was again not significant (Figure $\mathrm{S} 1(\mathrm{~b})$ ); when analysing over trials (mixed ANOVA), there was an effect of trial $(F(4.986,89.26)=3.258, p<0.01)$ and group $(F(1,18)=4.998, p<0.05)$ but no interaction and no pairwise by trial difference (Figure S1(c)). The group effect was probably due to a trend for more object visits in the scPCP group, particularly for later trials, but the absolute differences here were small. This again supports the view that scPCP rats were just as motivated as controls in their conNOR performance. Analysis of exploration time over the course of the conNOR protocol showed an effect of trial $(F(5.276,94.97)=12.33$, 


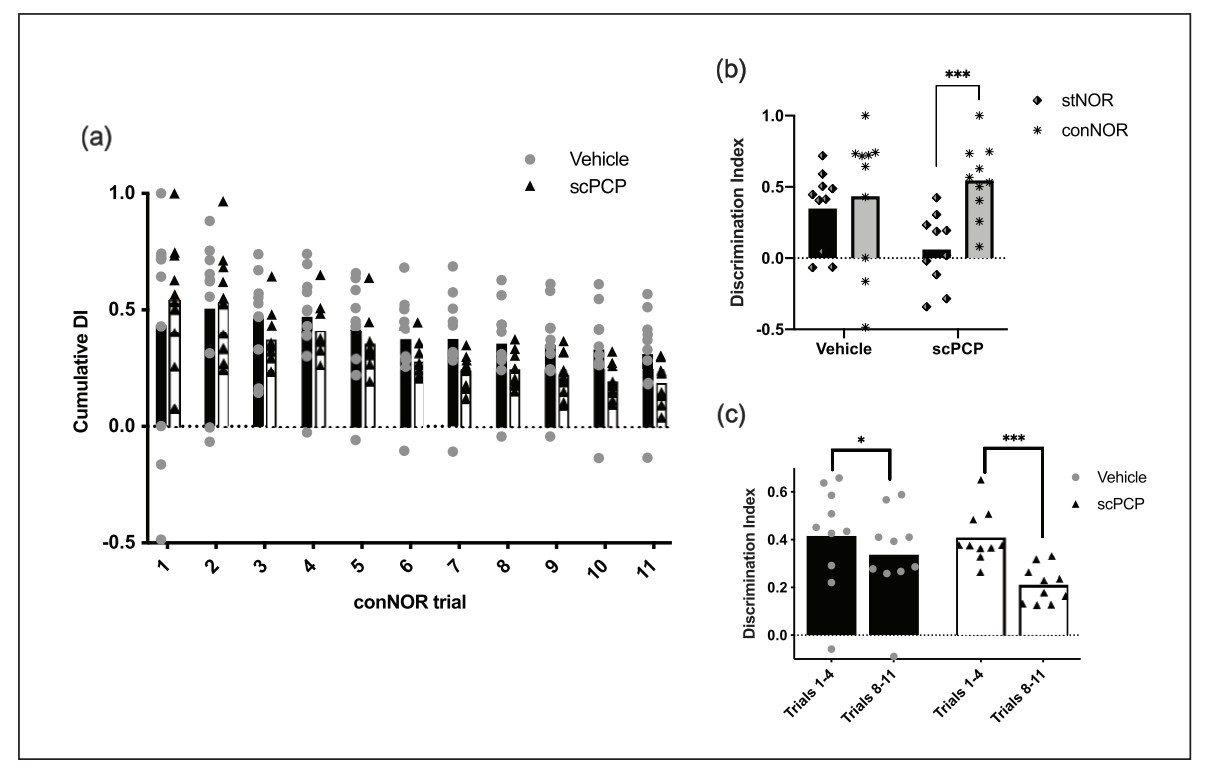

Figure 4. Continuous NOR (conNOR) performance in vehicle and scPCP rats. (a) Vehicle and scPCP groups show intact performance throughout the conNOR session, but SCPCP performance declines compared to vehicle group from trial 3, followed by a more consistent lower mean value from trial 7. (b) The stNOR deficit in SCPCP rats and their intact performance on conNOR trial 1 support the specific effect of distraction in this group for object memory in stNOR but not conNOR. (c) Decrease in performance for SCPCP rats over trials without distraction in conNOR is more substantial compared to vehicle. This supports an increased sensitivity of the SCPCP group to proactive interference compared to the vehicle group in conNOR. $N=10$ for both vehicle and SCPCP groups in all panels with their data presented as individual values plotted over group mean. ${ }^{*} p<0.05 ;{ }^{* * *} p<0.001$.

$p<0.001$ ), but no effect of treatment and no interaction (Figure S3(a)). There was no effect of PCP treatment on mean object pair exploration time (Figure S3(b)) and when comparing mean exploration times from the first versus the last four trials (Figure $\mathrm{S} 3(\mathrm{c}))$, there was a decline in exploration time between trial blocks $(F(1,6)=17.04, p<0.01)$ but no effect of treatment or interaction. Thus, although object exploration times tended to decrease and then plateau over conNOR trials, total object exploration for each trial was similar between vehicle and scPCP rats.

The rats' memory performance for conNOR is seen in the cumulative DI results, which show clearly that both scPCP and vehicle animals were able to recognise novelty throughout all 11 trials (Figure 4(a)). However, from trial 3 onwards, the mean performance of scPCP rats decreased and then plateaued at a lower DI level compared to vehicle. This pattern is supported by a significant effect of trial $(F(10,180)=13.48$, $p<0.001)$ and interaction between trial and treatment $(F(10,180)=2.33, \quad p<0.05)$ but no post hoc differences between scPCP and vehicle groups for any trial (Sidak). In addition, when considering initial versus late performance by averaging cumulative DI across the first versus the last fourtrial blocks (Figure 4(c)), there was an effect of trial block $(F(1,18)=43.20, p<0.01)$ and an interaction between treatment and trial block $(F(1,18)=8.013, p<0.05)$. Post hoc tests (Sidak) revealed significant differences between these fourtrial blocks for both vehicle $(p<0.05)$ and $\operatorname{scPCP}(p<0.001)$ treatment groups. Thus, while both groups showed good object memory throughout conNOR testing, there was a significant performance deficit in the scPCP group compared to vehicle that became more pronounced with increasing numbers of trials (proactive interference).

To determine whether we could reproduce the effect of distraction on the persistence of object memory after ScPCP treatment, we compared performance of stNOR (distraction) to that of conNOR trial 1 (no distraction; Figure 4(b)). These data show a clear pattern where vehicle rats perform well in both NOR versions whereas ScPCP rats show a specific deficit in stNOR only. Thus, there was an effect of the type of NOR test (stNOR vs conNOR; $F(1,18)=14.56, p<0.01)$ and an interaction between NOR test and treatment $(F(1,18)=7.155$, $p<0.05$ ). Post hoc (Sidak) tests showed that there was a significant difference between stNOR and conNOR performance for the scPCP group only $(p<0.001)$. In addition, apart from scPCP rats undergoing stNOR all other groups performed significantly above chance $(\mathrm{DI}=0$; one sample $t$-test; stNOR and conNOR for vehicle both $p<0.05$, conNOR scPCP $p<0.01$ ). These data clearly show that object memory deficits in scPCP rats are sensitive to both distraction and proactive interference and that these effects can be dissociated behaviourally using two-related tests of object preference - the stNOR and conNOR paradigms, respectively.

\section{Anatomical changes in the SCPCP mouse prefrontal cortex}

Brains from all animals were analysed for changes in the density of PV-positive cells in PFC (Figure 3(c) and (d)). There was a significant reduction for $\mathrm{scPCP}$ rats $(p<0.001$; two-tailed 
unpaired $t$-test) compared to vehicle, which agrees well with prior validation of the scPCP model.

\section{Discussion}

The acquisition and flexible use of new memory is susceptible to several disruptions of cognitive control. These include difficulty in maintaining attention on new information in the face of distraction and contagion by related, older memories when trying to recall newly encoded information (Postle and Brush, 2004; Postle et al., 2004). These sensitivities to distraction and proactive interference, respectively, are debilitating symptoms in a range of neuropsychiatric diseases; indeed, they are likely to be fundamental factors in underpinning a wide range of CIAS (Girard et al., 2018). CIAS are difficult to treat with current therapies (Keefe et al., 2007; Nuechterlein et al., 2004), so there is a pressing need for new drug discovery in this area of symptomatology. A core prerequisite for the latter is a range of preclinical models that show CIAS-related deficits in simple, high-throughput tasks that allow rapid testing of novel compounds while maintaining high translational validity. Currently, testing for cognitive control deficits in CIAS models requires training of rodents in complex operant tasks that often takes weeks to months before animals reach criterion prior to subsequent testing. While these tasks are reliable and offer high translational validity, they are low-throughput. Ideally, our preclinical armoury of tasks to investigate cognitive control aspects such as resistance to distraction and interference would also include high-throughput, simple, short-duration tasks that probe these aspects of cognition by instead relying on spontaneous behaviour. An obvious candidate here is the highly popular NOR paradigm, which takes advantage of the innate preference of rodents to explore novelty (Ennaceur and Delacour, 1988). Here, we tested whether disease-relevant sensitivity to distraction and proactive interference could be dissociated in variants of the NOR task using a rat model of CIAS. A successful outcome would provide powerful new options for future drug discovery programmes through simple, high-throughput task options, each sensitive to a selective aspect of memory impairment in schizophrenia.

Our chosen model was the subchronic phencyclidine (scPCP) treated rat, developed to model the $N$-methyl-D-aspartate (NMDA) receptor hypofunction hypothesis of schizophrenia (Coyle, 2006; Javitt and Zukin, 1991; Krystal et al., 1994; Steinpreis, 1996). The scPCP rat model effectively produces CIAS-associated neurobiological and cognitive impairments (Lisman et al., 2008; Neill et al., 2010, 2014). Thus, both rat and mouse scPCP models show a robust decrease in PV expression (Abdul-Monim et al., 2007; Gigg et al., 2020; Reynolds and Neill, 2016), consistent with inhibitory neurone changes in the cortex and hippocampus of schizophrenic patients (Beasley et al., 2002; Beasley and Reynolds, 1997; Benes et al., 1991). A defining feature of the scPCP model is a deficit in the stNOR test (Gigg et al., 2020; Hashimoto et al., 2008; Horiguchi and Meltzer, 2012; Nagai et al., 2009; Snigdha et al., 2010). scPCP performance is susceptible to task-related distraction during the stNOR inter-trial delay, supporting the conclusion that initial object memory encoding is good in scPCP rodents, at least over a single trial, but their ability to maintain this information is more sensitive to disruption compared to controls (Gigg et al., 2020; Grayson et al., 2014). Thus, studies using drugs to improve stNOR performance in the scPCP model in particular and other models more generally should take into account the possibility that effects may be via improving attention rather than modulating memory per se. While the present data agree with prior studies that scPCP rodents have no deficit in acquiring and maintaining object memory in the absence of distraction, we tested whether there was a quantitative difference in how scPCP rats use their whiskers to sense this information. Whisker movements during object contact are a vital source of environmental information for rodents, and these are abnormal in other rodent models of neuropsychiatric disease that express sensory, motor and cognitive deficits (Garland et al., 2018; Grant et al., 2014, 2020; Simanaviciute et al., 2020). We measured various aspects of whisker movement in vehicle- and PCP-treated rats and found no differences, supporting the view that object-touch-related information is preserved at the earliest stages of sensory input. We were confident that that the scPCP phenotype was established in these rats, demonstrated by their inability to discriminate novelty in the single trial NOR test and decreased density of PV-positive neurones within the medial PFC. Therefore, we suggest that the lack of whisker movement deficit following scPCP induction here may be due to using an adult rather than developmental model. Indeed, there is ample evidence for sensorimotor acquisition and integration disturbances in schizophrenic patients, potentially affecting the sense of self and body boundaries in particular (Postmes et al., 2014), and many transgenic preclinical models express changes in whisker movements and somatosensory cortical plasticity (Greenhill et al., 2015; Simanaviciute et al., 2020). Although whisker movements can be easily tracked and quantified to measure movement deficits, they are also relatively robust to neural changes (Garland et al., 2018; Grant et al., 2014, 2020), with forms of whisker movements always present, even in late-stage transgenic models. Nevertheless, at least from a behavioural perspective, the acquisition of whisker-related sensory input appears normal in the adult scPCP rat here. While this would support no effect of scPCP on object exploration by whisker movements in the stNOR task, we cannot make the same presumption for conNOR performance, as rats were food restricted in this phase. However, as both groups appeared equally engaged in the conNOR task (Figures S1-S3), this suggests that overt differences in whisker movements in conNOR would be unlikely.

We were next able to reproduce the documented sensitivity of stNOR performance to distraction in the scPCP model (Gigg et al., 2020; Grayson et al., 2014). This was determined by directly comparing stNOR performance to that of the first continuous NOR (conNOR) trial. The major difference between these variants of the same NOR paradigm is that stNOR includes inter-trial interval handling and removal to a holding age, whereas in conNOR, the rat is left to shuttle to the holding arena without any intervention from the experimenter (Ameen-Ali et al., 2012; Chan et al., 2018). Both NOR variants share initial handling into the apparatus for the acquisition phase with exploration of two identical novel objects. Thus, we were able to behaviourally dissociate the effects of distraction on memory persistence in the scPCP rat using two variants of the same basic NOR paradigm. Our next step was to determine whether the intact memory performance of 
scPCP rats after the first conNOR trial would continue across the remaining 10 continuous trials of the session. The data showed clearly that while both groups explored objects with similar visit frequency and total duration and showed a cumulative DI score that was above chance on each trial, scPCP performance declined significantly relative to controls from trial 3 onwards. This pattern of decreasing performance in the face of increasing, modalityspecific memory from prior trials strongly supports a proactive interference effect. Thus, the continuous accumulation of object memory in the scPCP group over trials was interfering strongly with their instantaneous judgement of object novelty. Of note, the same effect was seen but to a much-reduced extent in the vehicle group. While we used dietary restriction to promote conNOR performance (Chan et al., 2018), in practice many pellets were either not collected or left unconsumed by rats during the task, so we feel performance would be equally good in future studies without prior food restriction. Importantly, food restriction had no obvious differential effect on motivation for conNOR performance in scPCP rats, as seen by their similarity to controls in terms of latency to shuttle, pellet dispenser visits and time to complete the session. In addition, the similar duration and frequency of object encounters between groups strongly suggest that scPCP rats were not distracted during conNOR trials, further supporting the conclusion that their conNOR deficit was specific to proactive interference. Overall, these observations strongly support the presence of two separable deficits of cognitive control in the scPCP rat: distraction and proactive interference. The importance of this result is that two core cognitive symptoms of schizophrenia can be behaviourally dissociated in the scPCP CIAS model using two variants of the same simple, high-throughput NOR procedure. This dissociation would be strengthened further by, for example, future methodological changes to test rats purely in the conNOR apparatus in conditions either with or without distraction. This would eliminate any confound regarding the different arenas used here for stNOR and conNOR. Including a final stNOR trial would also be a useful means to ensure that the stNOR distraction effect persists after conNOR training.

It is worth considering the neural mechanisms that might underpin the present cognitive deficits in the scPCP model. There is substantial evidence that damage to PFC produces increased sensitivity to distraction and proactive interference. This region shows clear changes in schizophrenic patients with a strong parallel in functionally equivalent regions of frontal cortex in the scPCP model. While there is good evidence for PFC dysfunction in facilitating interference, there is also evidence that regions such as the perirhinal cortex (PRC) are important in this respect, particularly for object memory. Substantial evidence shows that PRC is vital for judgements of object familiarity and data from human functional magnetic resonance imaging (fMRI) also show increased PRC activity for object memory retrieval under conditions of object but not spatial proactive interference (Watson and Lee, 2013). This role for PRC in reducing object interference may be part of a wider medial temporal lobe network that includes lateral entorhinal cortex and hippocampus (Reagh and Yassa, 2014) that, in turn, communicate with PFC. These temporal lobe regions show decreased volume in schizophrenic patients and adolescents with a high risk of the disease (Roalf et al., 2017; Sim et al., 2006; Turetsky et al., 2003). Perirhinal lesions in wild-type rats produce a very similar pattern of proactive interference interfering with object memory over sequential trials in the bow-tie maze (Aggleton et al., 2010; Albasser et al., 2015) to that seen here in the conNOR performance of scPCP rats. In addition, a recent brain volume analysis in ScPCP rats showed that while there was a general shrinkage across all regions, this was particularly strong for PRC (Doostdar et al., 2019). Thus, the strong effect of proactive interference in conNOR described here for scPCP rats may be a particular function of PRC damage in the model, which would be consistent with lesion data and results from schizophrenic patients.

In summary, this study has shown a behavioural dissociation between effects of distraction and proactive interference on object memory by employing two variants of the same NOR paradigm in the scPCP model for CIAS. This provides a new set of high-throughput tasks with which to probe fundamental cognitive symptoms of schizophrenia in preclinical models.

\section{Acknowledgements}

The authors are very grateful to Mr Matthew Burgess for expert technical assistance with behavioural testing. We are also grateful to support from Durham University's Research Impact Fund for the continuous trials apparatus.

\section{Author contributions}

J.G., U.S., R.A.G. and M.H.H. contributed to the conceptualisation of the study. K.L., J.F. and U.S. contributed to data curation of the study. K.L., U.S., J.F. and J.G. contributed to the formal analysis of the study. K.L., U.S., J.F. and J.G. contributed to investigation of the study. K.L., J.F., J.G., U.S., R.A.G. and M.H.H. contributed to the methodology of the study. J.G., R.A.G., M.H.H. and B.G. contributed to the project administration of the study. B.G., M.H.H., R.A.G. and J.G. contributed to the resources of the study. U.S. and R.A.G. contributed to the software of the study. J.G., R.A.G. and M.H.H. contributed to the supervision of the study. J.G., K.L., J.F., B.G., U.S., R.A.G., B.G. and M.H.H. contributed to writing the original draft of the study. JG and M.H.H. contributed to the final writing, review and editing of the study.

\section{Declaration of conflicting interests}

The author(s) declared no potential conflicts of interest with respect to the research, authorship and/or publication of this article.

\section{Funding}

The author(s) received no financial support for the research, authorship and/or publication of this article.

\section{ORCID iD}

J. Gigg (iD) https://orcid.org/0000-0001-8253-1582

\section{Supplemental material}

Supplemental material for this article is available online.

\section{References}

Abdul-Monim Z, Neill JC and Reynolds GP (2007) Sub-chronic psychotomimetic phencyclidine induces deficits in reversal learning and alterations in parvalbumin-immunoreactive expression in the rat. Journal of Psychopharmacology 21(2): 198-205. 
Aggleton JP, Albasser MM, Aggleton DJ, et al. (2010) Lesions of the rat perirhinal cortex spare the acquisition of a complex configural visual discrimination yet impair object recognition. Behavioral Neuroscience 124(1): 55-68.

Albasser MM, Chapman RJ, Amin E, et al. (2010) New behavioral protocols to extend our knowledge of rodent object recognition memory. Learning \& Memory 17(8): 407-419.

Albasser MM, Olarte-Sanchez CM, Amin E, et al. (2015) Perirhinal cortex lesions in rats: Novelty detection and sensitivity to interference. Behavioral Neuroscience 129(3): 227-243.

Ameen-Ali KE, Eacott MJ and Easton A (2012) A new behavioural apparatus to reduce animal numbers in multiple types of spontaneous object recognition paradigms in rats. Journal of Neuroscience Methods 211(1): 66-76.

Barch DM, Braver TS, Carter CS, et al. (2009) CNTRICS final task selection: Executive control. Schizophrenia Bulletin 35(1): 115-135.

Beasley CL and Reynolds GP (1997) Parvalbumin-immunoreactive neurons are reduced in the prefrontal cortex of schizophrenics. Schizophrenia Research 24(3): 349-355.

Beasley CL, Zhang ZJ, Patten I, et al. (2002) Selective deficits in prefrontal cortical GABAergic neurons in schizophrenia defined by the presence of calcium-binding proteins. Biological Psychiatry 52(7): 708-715.

Benes FM, McSparren J, Bird ED, et al. (1991) Deficits in small interneurons in prefrontal and cingulate cortices of schizophrenic and schizoaffective patients. Archives of General Psychiatry 48(11): 996-1001.

Bondi MW, Monsch AU, Galasko D, et al. (1994) Preclinical cognitive markers of dementia of the Alzheimer type. Neuropsychology 8(3): 374-384.

Burton BK, Vangkilde S, Petersen A, et al. (2018) Sustained attention and interference control among 7-year-old children with a familial high risk of schizophrenia or bipolar disorder - A nationwide observational cohort study. Biological Psychiatry: Cognitive Neuroscience and Neuroimaging 3(8): 704-712.

Cadinu D, Grayson B, Podda G, et al. (2018) NMDA receptor antagonist rodent models for cognition in schizophrenia and identification of novel drug treatments, an update. Neuropharmacology 142: 41-62.

Chan M, Eacott MJ, Sanderson DJ, et al. (2018) Continual trials spontaneous recognition tasks in mice: Reducing animal numbers and improving our understanding of the mechanisms underlying memory. Frontiers in Behavioral Neuroscience 12: 214.

Coyle JT (2006) Glutamate and schizophrenia: Beyond the dopamine hypothesis. Cellular and Molecular Neurobiology 26(4-6): 365-384.

Diamond A (2013) Executive functions. Annual Review of Psychology 64(1): 135-168.

Doostdar N, Kim E, Grayson B, et al. (2019) Global brain volume reductions in a sub-chronic phencyclidine animal model for schizophrenia and their relationship to recognition memory. Journal of Psychopharmacology 33(10): 1274-1287.

Elvevag B and Goldberg TE (2000) Cognitive impairment in schizophrenia is the core of the disorder. Critical Reviews in Neurobiology 14(1): 1-21.

Ennaceur A and Delacour J (1988) A new one-trial test for neurobiological studies of memory in rats. 1: Behavioral data. Behavioural Brain Research 31(1): 47-59.

Ettinger U, Aichert DS, Wostmann N, et al. (2018) Response inhibition and interference control: Effects of schizophrenia, genetic risk, and schizotypy. Journal of Neuropsychology 12(3): 484-510.

Garland H, Wood NI, Skillings EA, et al. (2018) Characterisation of progressive motor deficits in whisker movements in R6/2, Q175 and Hdh knock-in mouse models of Huntington's disease. Journal of Neuroscience Methods 300: 103-111.

Gigg J, McEwan F, Smausz R, et al. (2020) Synaptic biomarker reduction and impaired cognition in the sub-chronic PCP mouse model for schizophrenia. Journal of Psychopharmacology 34(1): $115-124$.
Gillespie D, Yap MH, Hewitt BM, et al. (2019) Description and validation of the LocoWhisk system: Quantifying rodent exploratory, sensory and motor behaviours. Journal of Neuroscience Methods 328: 108440.

Gilmour G, Arguello A, Bari A, et al. (2013) Measuring the construct of executive control in schizophrenia: Defining and validating translational animal paradigms for discovery research. Neuroscience \& Biobehavioral Reviews 37(9 Pt B): 2125-2140.

Girard TA, Wilkins LK, Lyons KM, et al. (2018) Traditional test administration and proactive interference undermine visual-spatial working memory performance in schizophrenia-spectrum disorders. Cognitive Neuropsychiatry 23(4): 242-253.

Grant RA, Sharp PS, Kennerley AJ, et al. (2014) Abnormalities in whisking behaviour are associated with lesions in brain stem nuclei in a mouse model of amyotrophic lateral sclerosis. Behavioural Brain Research 259: 274-283.

Grant RA, Wong AA, Fertan E, et al. (2020) Whisker exploration behaviours in the $5 \mathrm{xFAD}$ mouse are affected by sex and retinal degeneration. Genes, Brain and Behavior 19(3): e12532.

Grayson B, Adamson L, Harte M, et al. (2014) The involvement of distraction in memory deficits induced by NMDAR antagonism: Relevance to cognitive deficits in schizophrenia. Behavioural Brain Research 266: 188-192.

Grayson B, Idris NF and Neill JC (2007) Atypical antipsychotics attenuate a sub-chronic PCP-induced cognitive deficit in the novel object recognition task in the rat. Behavioural Brain Research 184(1): 31-38.

Green MF (1996) What are the functional consequences of neurocognitive deficits in schizophrenia? The American Journal of Psychiatry 153(3): 321-330.

Greenhill SD, Juczewski K, De Haan AM, et al. (2015) Adult cortical plasticity depends on an early postnatal critical period. Science 349(6246): 424-427.

Hashimoto K, Ishima T, Fujita Y, et al. (2008) Phencyclidine-induced cognitive deficits in mice are improved by subsequent subchronic administration of the novel selective alpha7 nicotinic receptor agonist SSR180711. Biological Psychiatry 63(1): 92-97.

Heckers S, Curran T, Goff D, et al. (2000) Abnormalities in the thalamus and prefrontal cortex during episodic object recognition in schizophrenia. Biological Psychiatry 48(7): 651-657.

Horiguchi M and Meltzer HY (2012) The role of 5-HT1A receptors in phencyclidine (PCP)-induced novel object recognition (NOR) deficit in rats. Psychopharmacology 221(2): 205-215.

Javitt DC and Zukin SR (1991) Recent advances in the phencyclidine model of schizophrenia. The American Journal of Psychiatry 148(10): 1301-1308.

Kahn RS and Keefe RS (2013) Schizophrenia is a cognitive illness: Time for a change in focus. JAMA Psychiatry 70(10): 1107-1112.

Keefe RS, Bilder RM, Davis SM, et al. (2007) Neurocognitive effects of antipsychotic medications in patients with chronic schizophrenia in the CATIE Trial. Archives of General Psychiatry 64(6): 633-647.

Krystal JH, Karper LP, Seibyl JP, et al. (1994) Subanesthetic effects of the noncompetitive NMDA antagonist, ketamine, in humans. Psychotomimetic, perceptual, cognitive, and neuroendocrine responses. Archives of General Psychiatry 51(3): 199-214.

Lisman JE, Coyle JT, Green RW, et al. (2008) Circuit-based framework for understanding neurotransmitter and risk gene interactions in schizophrenia. Trends in Neurosciences 31(5): 234-242.

Nagai T, Murai R, Matsui K, et al. (2009) Aripiprazole ameliorates phencyclidine-induced impairment of recognition memory through dopamine D1 and serotonin 5-HT1A receptors. Psychopharmacology 202(1-3): 315-328.

Neill JC, Barnes S, Cook S, et al. (2010) Animal models of cognitive dysfunction and negative symptoms of schizophrenia: Focus on NMDA receptor antagonism. Pharmacology \& Therapeutics 128(3): 419-432.

Neill JC, Harte MK, Haddad PM, et al. (2014) Acute and chronic effects of NMDA receptor antagonists in rodents, relevance to negative 
symptoms of schizophrenia: A translational link to humans. European Neuropsychopharmacology 24(5): 822-835.

Nuechterlein KH, Barch DM, Gold JM, et al. (2004) Identification of separable cognitive factors in schizophrenia. Schizophrenia Research 72(1): 29-39.

Park S, Puschel J, Sauter BH, et al. (2003) Visual object working memory function and clinical symptoms in schizophrenia. Schizophrenia Research 59(2-3): 261-268.

Postle BR and Brush LN (2004) The neural bases of the effects of itemnonspecific proactive interference in working memory. Cognitive, Affective, \& Behavioral Neuroscience 4(3): 379-392.

Postle BR, Brush LN and Nick AM (2004) Prefrontal cortex and the mediation of proactive interference in working memory. Cognitive, Affective, \& Behavioral Neuroscience 4(4): 600-608.

Postmes L, Sno HN, Goedhart S, et al. (2014) Schizophrenia as a selfdisorder due to perceptual incoherence. Schizophrenia Research 152(1): 41-50.

Reagh ZM and Yassa MA (2014) Object and spatial mnemonic interference differentially engage lateral and medial entorhinal cortex in humans. Proceedings of the National Academy of Sciences of the United States of America 111(40): E4264-E4273.

Reynolds GP and Neill JC (2016) Modelling the cognitive and neuropathological features of schizophrenia with phencyclidine. Journal of Psychopharmacology 30(11): 1141-1144.
Roalf DR, Quarmley M, Calkins ME, et al. (2017) Temporal lobe volume decrements in psychosis spectrum youths. Schizophrenia Bulletin 43(3): 601-610.

Sim K, DeWitt I, Ditman T, et al. (2006) Hippocampal and parahippocampal volumes in schizophrenia: A structural MRI study. Schizophrenia Bulletin 32(2): 332-340.

Simanaviciute U, Ahmed J, Brown RE, et al. (2020) Recommendations for measuring whisker movements and locomotion in mice with sensory, motor and cognitive deficits. Journal of Neuroscience Methods 331: 108532.

Snigdha S, Horiguchi M, Huang M, et al. (2010) Attenuation of phencyclidine-induced object recognition deficits by the combination of atypical antipsychotic drugs and pimavanserin (ACP 103), a 5-hydroxytryptamine(2A) receptor inverse agonist. Journal of Pharmacology and Experimental Therapeutics 332(2): 622-631.

Steinpreis RE (1996) The behavioral and neurochemical effects of phencyclidine in humans and animals: Some implications for modeling psychosis. Behavioural Brain Research 74(1-2): 45-55.

Turetsky BI, Moberg PJ, Roalf DR, et al. (2003) Decrements in volume of anterior ventromedial temporal lobe and olfactory dysfunction in schizophrenia. Archives of General Psychiatry 60(12): $1193-1200$

Watson HC and Lee AC (2013) The perirhinal cortex and recognition memory interference. Journal of Neuroscience 33(9): 4192-4200. 